\title{
Tumores Benignos de Ovario en la edad Pediátrica y Adolescencia.
}

\section{Benign Ovarian Tumors in Pediatric and Adolescence.}

*Correspondencia: vicentico2712@hotmail.com Teléfono [593] 992491338

Conflicto de intereses: Los autores declaran no tener conflictos de intereses.

Fondos: Ver la página 116

Recibido: 06Febrero2019 Aceptado: 22 Julio 2019 Publicado: 30 Agosto 2019

Membrete bibliográfico: Salinas V, Fajardo G, Luna J, Luna V, Ramírez J, Moreno A. Tumores Benignos de Ovario en la edad Pediátrica y Adolescencia. Rev. Oncol. Ecu 2019;29(2):110-118. ISSN: 2661-6653

DOI: https://doi.org/10.33821/86

Copyright Salinas et al. Este artículo es distribuido bajo los términos de Creative Commons Attribution License 4.0 , el cual permite el uso y redistribución citando la fuente y al autor

\author{
Vicente Aníbal Salinas Salinas ${ }^{*}$ iD, Gabriela Yulissa Fajardo Ponce² \\ (iD), Julián Alfonso Luna Montalván ${ }^{3}$, Victoria Samanta Luna \\ Panimboza², Juan Alfredo Ramírez Pico4 ${ }^{i D}$, Andy Michael Moreno \\ Espinoza2
}

1. Universidad Católica de Santiago de Guayaquil (UCSG), Guayaquil, Ecuador, Postgrado de Cirugía Pediátrica.

2. Universidad Estatal de Guayaquil, Guayaquil, Ecuador, Postgrado de Cirugía Pediátrica.

3. Hospital Pediátrico Roberto Gilbert Elizalde, Servicio de Cirugía Pediátrica, Guayaquil-Ecuador.

4. Servicio de Unidad Pediátrica, SOLCA - Guayaquil.

\section{Resumen}

Introducción: Los tumores ováricos son raros en la edad pediátrica y representan del 1 al $5 \%$ de los tumores infantiles. El objetivo del presente trabajo es conocer cuáles son los tumores de ovario más frecuentes en nuestro medio en niñas y adolescentes en un centro oncológico de la ciudad de Guayaquil-Ecuador.

Métodos: El presente estudio es descriptivo y retrospectivo, en la que se realiza una revisión de registros de niñas y adolescentes con diagnóstico de tumores de ovario, en el Instituto Oncológico Nacional "Dr. Juan Tanca Marengo" en el período de enero 2015 a mayo del 2017. Las variables registradas fueron edad, diagnóstico ecográfico, diagnostico histopatológico, ovario afecto, características clínicas, salpingectomía, marcadores tumorales. Se utiliza estadística descriptiva.

Resultados: Ingresaron al estudio 32 pacientes de 12.7 años promedio. El ovario más afectado fue el derecho con una relación 2.2: 1 y en $9 \%$ fue bilateral. La histología reportada de los quistes serosos fueron el $21 \%$ quiste folicular y $18.5 \%$ por quiste lúteo. Los quistes simples representaron el $15.25 \%$, elcistoadenoma y el teratoma maduro con un $9 \%$, el quiste dermoide y le endometrioma con un $3 \%$. El dolor fue el síntoma más llamativo estado como síntoma único en $65.5 \%$. El $18.75 \%$ terminó en anexectomía con ooforectomía por extensión del quiste, el 81.25 \% solo requirió quistectomía.

Conclusión: La histología reportada en esta serie de tumores benignos de ovario, los quistes serosos foliculares fueron los más prevalentes, seguidos de los quistes lúteos. El dolor pélvico fue el síntoma más llamativo. La mayoría de los casos requirieron quistectomía y el $19 \%$ requirió adicionalmente ooforectomía.

Palabras Claves: ENFERMEDADES DEL OVARIO, NEOPLASIAS OVÁRICAS, PEDIATRÍA. 
DOI: https://doi.org/10.33821/86

\section{Abstract}

Introduction: Ovarian tumors are rare in pediatric age and represent 1 to $5 \%$ of childhood tumors. The objective of this work is to know which are the most frequent ovarian tumors in our environment in girls and adolescents in an oncological center of the city of Guayaquil-Ecuador.

Methods: The present study is descriptive and retrospective, in which a review of records of girls and adolescents diagnosed with ovarian tumors is performed, at the National Oncology Institute "Dr. Juan Tanca Marengo" in the period from January 2015 to May 2017. The variables recorded were age, ultrasound diagnosis, histopathological diagnosis, affected ovary, clinical characteristics, salpingectomy, tumor markers. Descriptive statistics are used.

Results: 32 patients with an average age of 12.7 entered the study. The most affected ovary was the right one with a 2.2: 1 ratio and in $9 \%$ it was bilateral. The reported histology of serous cysts were $21 \%$ follicular cyst and $18.5 \%$ by luteal cyst. Simple cysts accounted for $15.25 \%$, cystadenoma and mature teratoma with $9 \%$, dermoid cyst and endometrioma with $3 \%$. Pain was the most striking condition as a single symptom in $65.5 \% .18 .75 \%$ ended up in anexectomy with oophorectomy due to cyst extension, $81.25 \%$ only required a cystectomy.

Conclusion: The histology reported in this series of benign ovarian tumors, follicular serous cysts were the most prevalent, followed by luteal cysts. Pelvic pain was the most striking symptom. The majority of cases required a cystectomy and $19 \%$ additionally required oophorectomy.

Keywords: OVARY DISEASES, OVARIAN NEOPLASIAS, PEDIATRICS.

\section{Introducción}

Los tumores ováricos son raros en la edad pediátrica y representan del 1 al $5 \%$ de los tumores infantiles. Son más frecuentes entre los 9 y los 12 años [1, 2].

En los niños $68 \%$ de los tumores son por células germinales y $16 \%$ por células epiteliales, Lafrecuencia de neoplasias malignas de ovario es de $3.4 \%$, pues la mayor parte de los tumoresdel ovario son benignos; los más comunes son los del cuerpo lúteo, quistes simples, quistesfoliculares, quistes paraováricos y lesiones inflamatorias [3, 4].

Los quistes paratubáricos o paraováricos son de diagnóstico infrecuente en la edad pediátrica. Suelen ser quistes simples de pequeño tamaño que aparecen sobre el ligamento ancho o sobre la propia trompa de Falopio. Derivan de tejido mesotelial, aunque en algunas ocasiones pueden provenir de restos embrionarios, normalmente del paramesonefros $\mathrm{y}$, rara vez del mesonefros [5].

De todos los quistes ováricos, se describe que al menos el $90 \%$ se resuelve en forma espontánea. Se recomienda manejo conservador con seguimiento ultrasonográfico, al menos durante tres ciclos. Los quistes ováricos funcionales son parte normal del desarrollo folicular durante el ciclo menstrual. Se pueden formar quistes patológicos, que habitualmente son de aspecto simple, porque un folículo no ovuló, ni involucionó o bien después de la ovulación, 
pueden existir quistes funcionales que se originan del cuerpo lúteo, los cuales sangran con facilidad, por lo que su aspecto es complejo y pueden alcanzan tamaños de 6 a $8 \mathrm{~cm}$ [6].

Aunque la mayoría de las masas anexiales son benignas, el objetivo principal en la evaluación diagnóstica es excluir la posibilidad de que se trate de un proceso maligno. Se estima entre 4-6 \% la probabilidad de encontrar malignidad en una masa anexial de aspecto no maligno [7].

El diagnóstico se basa en las pruebas de imagen (ecografía y TC fundamentalmente), los marcadores tumorales y el estudio anatomo-patológico. Destaca la gran sensibilidad de la ecografía, mayor que la de la TC para algunos autores [8].

El objetivo del presente trabajo es conocer cuáles son los tumores de ovario más frecuente en nuestro medio.

\section{Materiales y Métodos}

El presente estudio es de tipo descriptivo, diseño no experimental, retrospectivo. Consiste enuna revisión de registros de pacientes con diagnóstico de tumores de ovario (masa anexial) de mujeres en edad pediátrica y adolescentes de 3 a 17 años, en el Instituto Oncológico Nacional "Dr. Juan Tanca Marengo" en el período de enero 2015 a mayo del 2017.

Se excluyeron registros de pacientes que tuvieron los datos incompletos.

Los datos fueron obtenidos de las historias clínicas, y a través de un programa de recolección de datos diseñado por data editor de Microsoft (SPSS 13.0) se analizaron las variables: edad, diagnóstico ecográfico, diagnostico histopatológico, ovario afecto, características clínicas, salpingectomía, marcadores tumorales.

Los datos se presentan en frecuencias relativas y absolutas, por edad y de subtipos histológicos corroborados por patología. Se realizó el estudio con la aprobación del Departamento de Docencia e Investigación, y por las autoridades de Solca.

Las características de la población y los hallazgos del estudio se presentaron a través de estadígrafos, histogramas, porcentajes. 


\section{Resultados}

Ingresaron al estudio 32 pacientes. La media de edad fue de 12.7 años, con un rango de 3 a 17 años. La frecuencia de casos aumentó con la edad: en el grupo de edad de 3 a 7 años se presentaron 4 casos (12\%), en el grupo de 8 a 12 años 10 casos (32\%) y en el grupo de 13 a17 años fueron 18 casos (56\%) (Figura 1).

Figura 1. Grupos de edad de pacientes con tumores de ovario

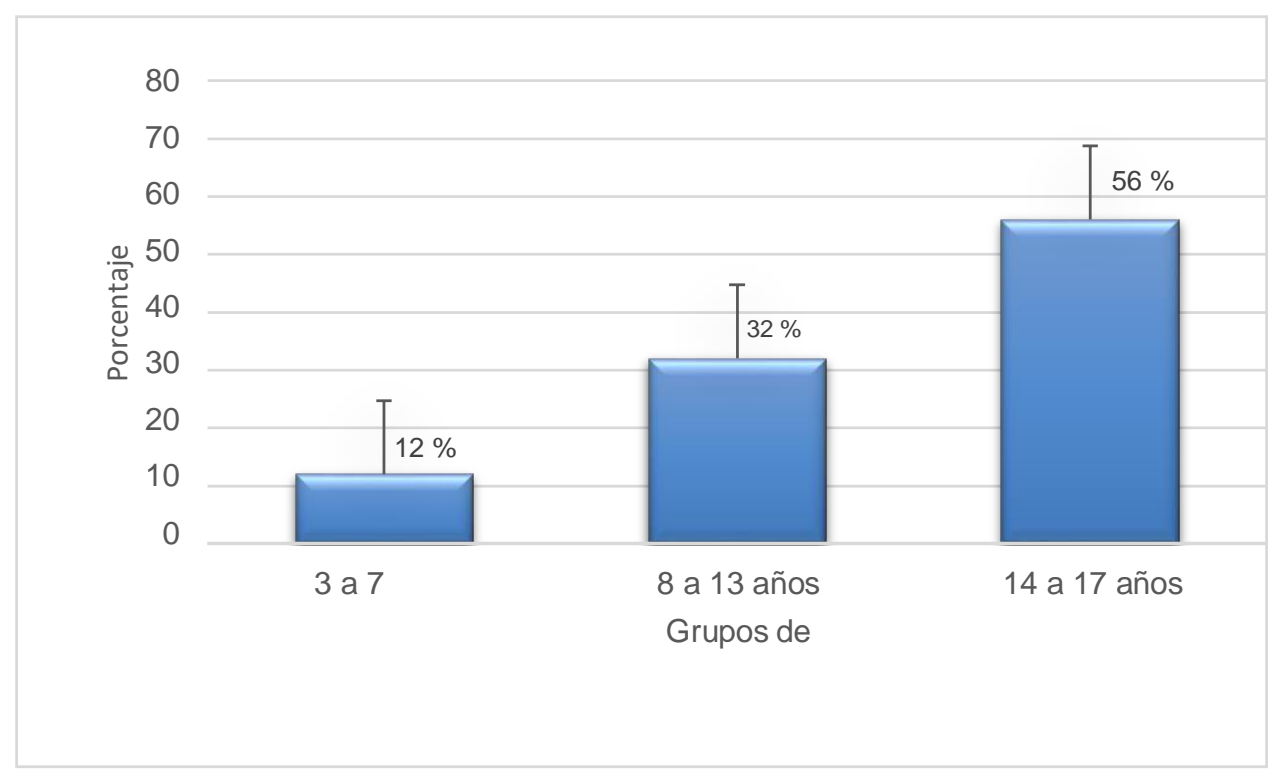

En un $96 \%$ de los pacientes se realizó ecografía ayudando a la decisión terapéutica y el tipo de abordaje. El ovario más afectado fue el derecho con una relación 2.2: 1 con el izquierdo y el $9 \%$ fue bilateral (Figura 2).

Figura 2. Anexo Afectado diagnosticado por ecografía.

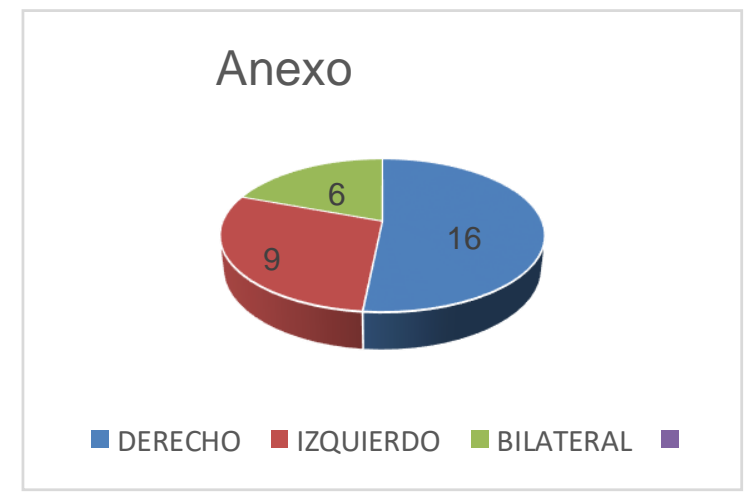

La histología reportada de los quistes serosos fueron el $21 \%$ quiste folicular y $18.5 \%$ por quiste lúteo. Los quistes simples representaron el $15.25 \%$, el cistoadenoma y el teratoma maduro con un $9 \%$, el quiste dermoide y le endometrioma con un $3 \%$ (Figura 3 ). 
Figura 3. Tipos de tumores benignos

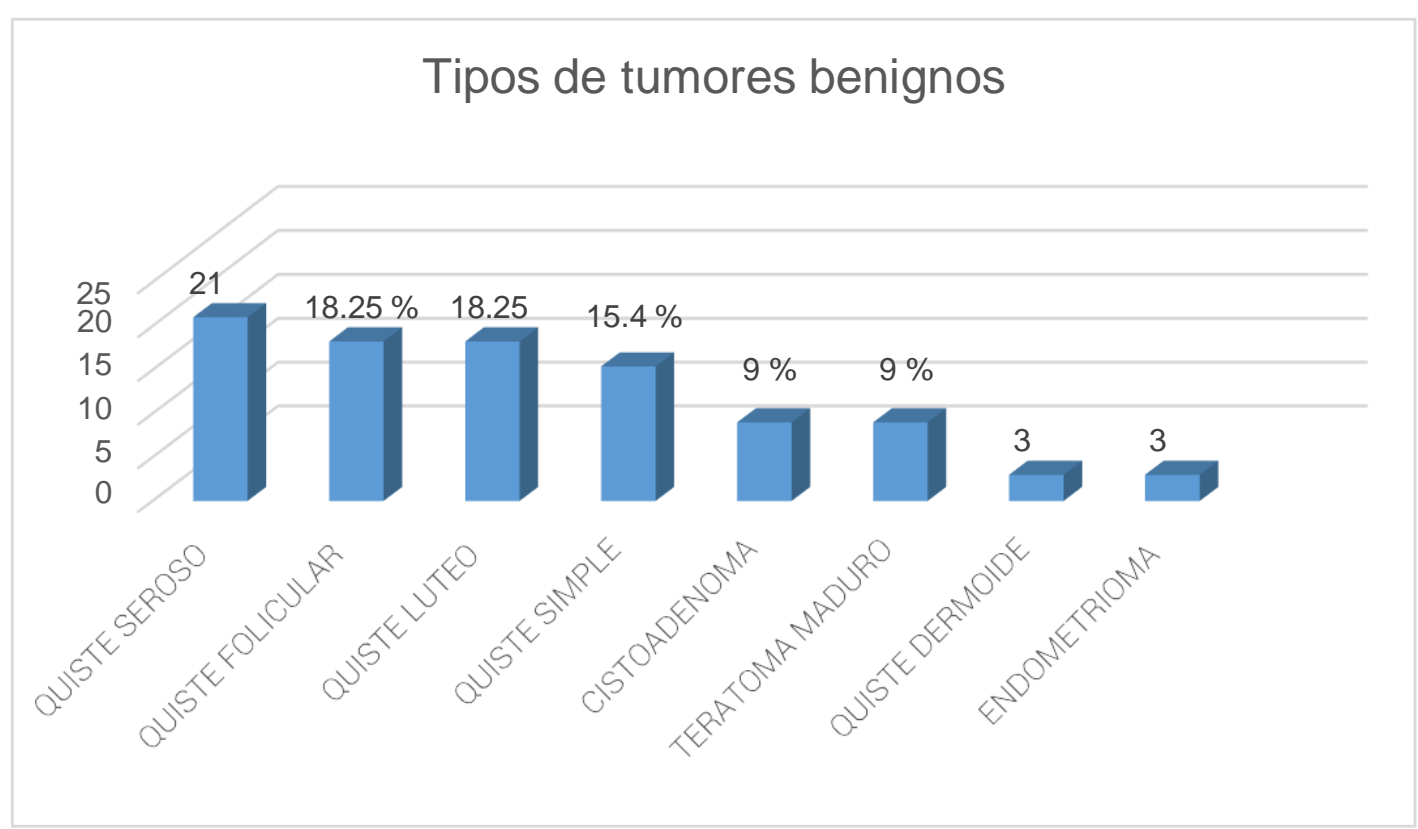

El dolor fue el síntoma más llamativo estado como síntoma único en $65.5 \%$, el vómito represento el $6 \%$, la combinación de ambos $25 \%$, solo el $3.5 \%$ se presentó como una masa palpable asintomática. El $18.75 \%$ terminó en anexectomía con ooforectomía por extensión del quiste, el 81.25 \% solo requirió quistectomía (Figura 4).

Figura 4. Tipo de intervención quirúrgica.

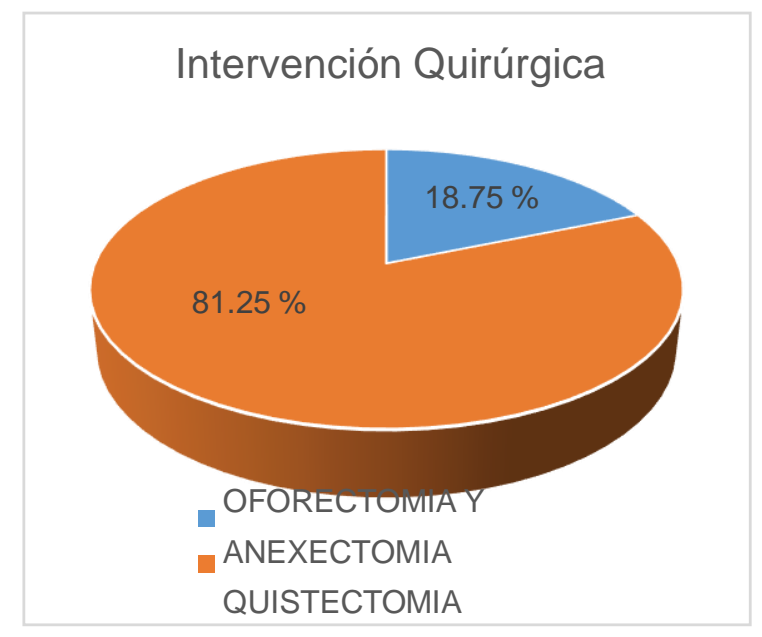

En los pacientes con sospecha de malignidad se realizó marcadores tumorales que representaron el 59 \% de la población en estudio, en quienes fueron negativos.

En el 100 \% de la población en estudio la resolución fue laparoscópica. 


\section{Discusión}

En un estudio de 1818 niñas menores de 8 años que se realizaron ecografías por otros motivos se encontró una incidencia de 2 a $3 \%$ de quistes ováricos funcionales [6], en presente reporte el quiste seroso fue el más frecuente representando $21 \%$.

La complicación más frecuente es la torsión (50-78 \%), esto debido a que el pedículo infundíbulo pélvico de las niñas es mayor que el de la mujer adulta, lo que facilita la torsión del ovario aumentado de tamaño, que se acompaña de dolor característico [4]. El dolor fue elsigno característico en el presente estudio con un $65 \%$ de prevalencia.

Otras complicaciones son obstrucción o perforación gastrointestinal, obstrucción del tracto urinario, ruptura del quiste, hemorragia, necrosis, pérdida de la gónada, se ha mencionado además en forma aislada complicaciones endocrinas como hipoglicemia, hipotiroidismo e hipocalcemia [9].

Por el riesgo de malignidad en la edad prepuberal, en los quistes complejos se recomienda su resección más que observar. También tienen indicación quirúrgica las pacientes sintomáticas en que se sospecha torsión de ovario y los quistes simples que no regresan en control de 1 a 6 meses; considerando que generalmente un quiste funcional regresa o se modifica en 1 a 2 semanas. El uso de hormonas está indicado principalmente para inhibir la aparición de otros quistes, pero no actúa sobre el quiste ya formado [10].

El uso de los marcadores tumorales permite tener una orientación en cuanto a la posibilidad de malignidad de una masa anexial [11]. Al 59 \% de la población en este estudio se realizó marcadores tumorales.

El tratamiento quirúrgico está reservado para pacientes que presentan síntomas o signos que permitan sospechar torsión o malignidad [10].

Lo que ocurre es que existe riesgo de malignización (2.9\%) y se discute si la vía laparoscópica es apta para estos casos. En casos en los que se dispone de tiempo para completar la historia clínica con marcadores tumorales y pruebas de imagen, la técnica de elección es la laparoscopia, siempre que la sospecha de malignidad sea escasa. Pero si existen dudas normalmente esta técnica debe ser descartada [12].

La laparoscopia reduce el dolor postoperatorio, disminuye la creación de adherencias, favorece el alta precoz y produce un mejor resultado estético [13]. El $100 \%$ de los participantes en este reporte fueron tratados por esta vía.

La posibilidad de hallar incidentalmente en la anatomía patológica, un proceso neoplásico maligno en una cirugía laparoscópica por tumoración anexial, oscila entre 0.4 a 2.9 \% [14]. Eneste estudio no se reportaron tumores malignos. 


\section{Conclusiones}

La histología reportada en esta serie de tumores benignos de ovario, los quistes serosos foliculares fueron los más prevalentes, seguidos de los quistes lúteos. El dolor pélvico fue el síntoma más llamativo. La mayoría de los casos requirieron quistectomía y el 19 \% requirió adicionalmente ooforectomía por extensión del quiste, por vía laparoscópica. En el 59 \% de los casos se realizaron marcadores tumorales los cuales fueron negativos.

\section{Agradecimientos}

Se reconoce a las personas que participaron indirectamente en el estudio tales como los pacientes, como personal técnico, otras en general de ION "Dr. Juan Tanca Marengo" SOLCA-Guayaquil.

\section{Información adicional}

\section{Abreviaturas}

TC: Tomografía

SOLCA: Sociedad de Lucha contra el Cáncer.

Nota del Editor

\section{La Revista Oncología Ecu permanece neutral con respecto a los reclamos jurisdiccionales en mapas publicados $y$ afiliaciones institucionales.}

\section{Archivos Adicionales}

Ninguno declarado por los autores.

\section{Fondos}

Los fondos para la presente investigación fueron propios de los autores del presente artículo.

\section{Disponibilidad de datos y materiales}

Existe la disponibilidad de datos bajo solicitud al autor de correspondencia. No se reportan otros materiales.

\section{Contribuciones de los autores}

VASS, GYFP, JALM, VSLP, JARP, AMME contribuyeron igual en el proceso de la investigación, idea de investigación, diseño, recolección de datos, análisis estadístico, escritura académica. El análisis crítico del artículo lo realizó AMME y JARP. Todos los autores leyeron y aprobaronla versión final del manuscrito. 


\section{Aprobación de ética y consentimiento para participar}

No aplica ya que es un estudio observacional retrospectivo.

\section{Consentimiento para publicación}

No aplica ya que es un estudio observacional retrospectivo.

\section{Información de los autores}

Vicente Aníbal Salinas Salinas (iD, Universidad Católica de Santiago de Guayaquil (UCSG), Guayaquil, Ecuador, Médico Residente del Postgrado de Cirugía Pediátrica.

Gabriela Yulissa Fajardo Ponce, Universidad Estatal de Guayaquil, Guayaquil, Ecuador, Médico Residente del Postgrado de Cirugía Pediátrica.

Julián Alfonso Luna Montalván, Hospital Pediátrico Roberto Gilbert Elizalde, Médico tratante del Servicio de Cirugía Pediátrica, Guayaquil-Ecuador.

Victoria Samanta Luna Panimboza, Universidad Estatal de Guayaquil, Guayaquil, Ecuador, Médico Residente del Postgrado de Cirugía Pediátrica.

Juan Alfredo Ramírez Pico, Instituto Oncológico Nacional "Dr. Juan Tanca Marengo" Solca, Guayaquil. Médico tratante del Servicio de Oncohematología.

\section{Referencias}

1. Brown M, Hebra A, McGeehin K, Ross A. Ovarian masses in children: A review of 91 cases of malignant and benign masses. J Pediatr Surgery 1993; 28:930-933.

2. Castleberry R, Cushing B, Perlman E, Hawkins E. Germ cell tumors. Principles and Practice of Pediatric Oncology: 3rd edition. Lippincott Co. Philadelphia 1997: 921-945

3. Meyer J, Harmon C. Harty P, Markowitz R, et al. Ovarían Torsión: Clinical and Imaging Presentation in Cnildren Journal of Pediatric Surgery, Vol 30, $N^{\circ} 10$ (October), 1995:1433-1436.

4. James L, Breen M, Bonamo F, Wayne S, Maxsen M, Genital tract in children. Pediatr. Clin. North. Am. 28: 355,1981

5. Schultz K, Ness K, Nagarajan R, Steiner M. Adnexal Masses in Infancy and Childhood. Clin Obstet Gynecol. 2006;49:464-79

6. Millar D, Blake J, Stringer D, Hara H, Babiak C. Prepubertal ovarian cyst formation: 5 years' experience. Obstet Gynecol 1993; 81: 434-438 
Abreviaturas en la referencias

\section{DOI: Digital Object}

Identifier

PMID: PubMed Identifier

SU: Short URL
7. Lehner R, Wenzl R, Heinzl H, Husslein P, Sevelda P. Influence of delayed staging laparotomy after laparoscopic removal of ovarian masses later found malignant. Obstet Gynecol 1998; 92(6): 967-71.

8. Pizzo P, Poplack D. Principles and Practice of Pediatric Oncology: 3rd edition. Lippincott Co. Philadelphia 1997: 921-945.

9. Brandt M, Luks F, Filiatrault D. Surgical indications in antenatally diagnosed ovarían cysts. J Pediatr Surg 1991;26:276-282.

10. Brandt M, Helmrath M. Ovarian cysts in infants and children. Semin Pediatr Surg 2005;14:78-85.

11. Nezhat C. Operative endoscopy will replace almost all open procedures. J Laparoendoscopic Surg 2004;8: 101-2.

12. Okada $\mathrm{T}$, Yoshida H, Matsunaga $\mathrm{T}$, Kouchi K, Ohtsuka $\mathrm{Y}$, Takano H, et al. Paraovarian cyst with torsion in children. J Pediatr Sur. 2002;37:937-40.

13. Pados G, Tsolakidis D, Bontis J. Laparoscopic management of the annexal mass. Ann N Y Acad Sci. 2006;1092:211-28.

14. Wenzl R, Lehner R, Husslein P, Sevelda P. Laparoscopic surgery in cases of ovarian malignancies: an Austria-wide survey. Gynecol Oncol 1996; 63(1): 57-61. 